T. Kurogi

Nagoya Math. J.

Vol. 95 (1984), 91-102

\title{
ISOMETRY INVARIANT CLOSED GEODESIC ON A NONPOSITIVELY CURVED MANIFOLD
}

\author{
TETSUNORI KUROGI*
}

\section{§. Introduction}

In this paper we wish to study the isometry invariant geodesic on a non-positively curved manifold from a point of view of the displacement function.

For an isometry $f$ on a compact connected Riemannian manifold $M$ a geodesic $c$ is called $f$-invariant geodesic if $f(c(t))=f(c(t+1))$ for any $t \in R$. And one point geodesic is called a trivial geodesic. (This is also a fixed point of $f$.) The isometry invariant geodesic was introduced by K. Grove ([5]) who studied it by using the infinite dimensional critical point theory and the Gromoll-Meyer type theorem was proved by Grove and Tanaka (cf. [7], [11], [12]). At the same time the good results were obtained for $\pi_{1}(M)=1$ (cf. [3], [6]). However for $\pi_{1}(M) \neq 1$ the mathematical phenomena for such a geodesic is not the same as the above case (cf. [1], [4], [6]). Here our method is different from their case because our manifold is topologically very simple but $\pi_{1}(M) \neq 1$. Our motivation comes from the works of T. Sunada (cf. [9], [10]) and V. Ozols ([8]).

In this paper we always assume that $M$ is a compact connected manifold with nonpositive sectional curvature. Let $F(f)$ be the fixed point set of an isometry $f$ on $M$. For the existence problem of such a geodesic the case of $\# F(f)<\infty$ (finite fixed points) is essential because (1) if $F(f)$ $=\phi$, there always exists such a geodesic $(2)$ if $\operatorname{dim}(F(f)) \geqq 1$, then $F(f)$ is a totally geodesic submanifold of $M$ which implies the existence (in particular it is a $f$-fixed geodesic).

Thus our first main result is as follows

Existence Criterion Theorem. Let $f$ be an isometry of finite order

Received May 25, 1983.

*) The work was done while the author was a Research Fellow at Nagoya University in 1982 supported by Japan Ministry of Education. 
$k(\geqq 1)$, let $F(f)=\left\{p_{1}, p_{2}, \cdots, p_{r}\right\}$ be the set of the finite fixed points of $f$ and $G_{p_{i}}=\left\{\mu \circ \tilde{f}_{i} \circ \mu^{-1} \mid \mu \in \Gamma\right\}$ where a covering isometry $\tilde{f}_{i}$ of $f$ has a fixed point on the fibre of $p_{i}$ and $\Gamma=\pi_{1}(M)$, then the following statements are mutually equivalent.

(1) There does not exist a non-trivial f-invariant geodesic.

(2) $\tilde{f}^{k}=1$ for any covering isometry $\tilde{f}$ of $f$.

(3) The set of the covering isometries of $\tilde{f}$ is just $\cup_{i=1}^{r} G_{p_{i}}$.

Since $f$ has the finite order $k$, every non-trivial $f$-invariant geodesic is closed. This assumption is not so special because the Bochner's theorem implies that every isometry has a finite order under the condition of the negative Ricci curvature.

Now let Geo $(M, f)$ be the set of the $f$-invariant closed geodesics. Then in a natural sense Geo $(M, f)=\bigcup_{r \in \Gamma} \mathfrak{g}_{[r]}^{f}$ where $\mathfrak{g}_{[r]}^{f}$ is the set of the free homotopic closed geodesic corresponding to the conjugate class [ $\gamma]$ for $\gamma \in \Gamma$. And let $\operatorname{Crit}(\tilde{f})$ be the set of $d_{\tilde{f}}^{2}$-critical points for the distance function $d_{\tilde{f}}(x)=d(x, \tilde{f}(x))$ for an isometry $\tilde{f}$ on $\tilde{M}$ which is the universal covering space of $M$. For any covering isometry $\tilde{f}$ of $f$ with $f^{k}=1$ we have $\tilde{f}^{k}=\gamma$ for some $r \in \Gamma$ and so let $G_{r}$ be the set of the covering isometries $\tilde{f}$ with $\tilde{f}^{k}=\gamma$. Now we define an equivalence relation on $G_{r}$ such that $\tilde{f} \sim \tilde{g}$ for any $\tilde{f}, \tilde{g}$ in $G_{r}$ if and only if there exists $\xi \in \Gamma_{r}$ with $\tilde{g}=\xi \circ \tilde{f} \circ \xi^{-1}$. We write the equivalence class of $\tilde{f}$ by $\langle\tilde{f}\rangle$.

StRUCTURe TheOREM Let $f$ be an isometry of finite order. Then $\mathfrak{g}_{[r]}^{f}$ is homeomorphic to $\bigcup_{\langle\tilde{f}\rangle} \operatorname{Crit}(\tilde{f}) \mid \Gamma_{\tilde{f}}$ where $\tilde{f} \in G_{\gamma}$ and $\Gamma_{\tilde{f}}=\{\gamma \in \Gamma \mid \gamma \circ \tilde{f}=\tilde{f} \circ \gamma\}$. Thus we have $\operatorname{Geo}(M, f)$ is homeomorphic to $\bigcup_{r \in \Gamma} \bigcup_{\langle j\rangle} \operatorname{Crit}(\tilde{f}) / \Gamma_{\tilde{f}}$. Moreover the above homeomorphism is a diffeomorphism if each $\operatorname{Crit}(\tilde{f})$ is a submanifold without boundary.

This structure theorem is an extension of the Sunada's one ([10]) to the case of $Z_{k}$-action. Moreover we have the similar theorem for a general isometry $f$ in Section 2.

And Section 3 provides an interesting example.

The author would like to thank Professor Y. Shikata for his constant encouragement with the author's whole heart. And the author is grateful to Professor T. Sunada and Professor M. Tanaka for many valuable discussions and comments. 


\section{§1. Existence}

Let $M$ be a compact connected manifold with nonpositive sectional curvature and $f$ be an isometry of $M$. Here we use the following notations.

$\tilde{M}$ : the universal covering space of $M, \pi: \tilde{M} \rightarrow M$ is the canonical projection.

$\tilde{f}$ : a covering isometry of $f$.

$\operatorname{Crit}(\tilde{f})$ : the set of all critical points of $d_{\tilde{f}}^{2}(x)=d^{2}(x, \tilde{f}(x))$ where $d$ is the Riemannian distance function on $\tilde{M}$.

$F(\tilde{f})$ : the fixed point set of $\tilde{f}$.

Note that $F(\tilde{f}) \subset \operatorname{Crit}(\tilde{f})$. The following theorem was proved by Ozols ([8]).

Lemma 1 (Ozols's theorem).

(1) $x \in \operatorname{Crit}(\tilde{f})-F(\tilde{f})$ if and only if $\tilde{f}$ preserves the minimizing geodesic from $x$ to $\tilde{f}(x)$.

(2) If $F(\tilde{f}) \neq \phi$, then $\operatorname{Crit}(\tilde{f})=F(\tilde{f})$.

(3) If $\xi: \tilde{M} \rightarrow \tilde{M}$ is an isometry, then $\operatorname{Crit}\left(\xi \circ \tilde{f} \circ \xi^{-1}\right)=\xi(\operatorname{Crit}(\tilde{f}))$.

If $c$ is an $f$-invariant geodesic, then there exists a lifted geodesic $\tilde{c}$ which is $\tilde{f}$-invariant for some covering isometry $\tilde{f}$, conversely if $\tilde{c}$ is an $\tilde{f}$-invariant geodesic for some covering isometry $\tilde{f}$, then the projection $\pi \circ \tilde{c}$ is $f$-invariant. Thus the above (1) suggests us to look into Crit $(\tilde{f})$ for the existence of the $f$-invariant geodesic. Of course since $\tilde{M}$ is noncompact $\operatorname{Crit}(\tilde{f})=\phi$ is possible. However we can prove the following lemma.

Lemma 2. Let $f$ be an isometry of $M$, then $\operatorname{Crit}(\tilde{f}) \neq \phi$ for any covering isometry $\tilde{f}$ of $f$.

Proof. For any covering isometry $\tilde{h}$ we prove that $d_{\tilde{h}}(x)=d(x, \tilde{h}(x))$ has a minimum on $\tilde{M}$. Without loss of generality we can assume $\inf d_{\tilde{h}}$ $=0$, if not we consider $d_{\tilde{h}}-a$ where $a=\inf d_{\tilde{h}}>0$. Let $\left\{x_{n}\right\} \subset \tilde{M}$ be a sequence such that $\lim _{n \rightarrow \infty} d_{\tilde{n}}\left(x_{n}\right)=\inf d_{\tilde{h}}=0$. For a fixed $x \in \tilde{M}$ and for the number $r=2$ (the diameter of $M$ ) the set $\left\{x \in \tilde{M} \mid d\left(x, x_{0}\right) \leqq r\right\}$ is compact. And so we can find $\gamma_{n} \in \Gamma$ such that $d\left(\gamma_{n}\left(x_{n}\right), x_{0}\right)<r$ for every $n \geqq 1$, thus $\left\{\gamma_{n}\left(x_{n}\right)\right\}$ has a limit point, say $y$. For a sufficiently large $n, d\left(\tilde{h}\left(x_{n}\right), x_{n}\right)$ $=d\left(\left(\gamma_{n} \tilde{h} \gamma_{n}^{-1}\right) \gamma_{n}\left(x_{n}\right), \gamma_{n}\left(x_{n}\right)\right)$ is near $\inf d_{\tilde{h}}=0$ and so $\gamma_{n} \tilde{h} \gamma_{n}^{-1}(y)$ is contained 
in a sufficient small neighborhood of $y$. On the other hand for any given $b>0$ and for any fixed $x \in \tilde{M}$, the set $\{\tilde{g} \mid \tilde{g}$ is a covering isometry of $f$ such that $d(x, \tilde{g}(x)) \leqq b\}$ is a finite set because $\tilde{g}$ is a covering isometry. Thus there exists $\gamma_{0} \in \Gamma$ such that $\gamma_{n} \tilde{h} \gamma_{n}^{-1}(y)=\gamma_{0} \tilde{h} \gamma_{0}^{-1}(y)$ for infinitely many $n$. Then $\inf d_{\tilde{h}}$ is attained at $\gamma_{0}^{-1}(y)$. This implies Crit $(\tilde{h}) \neq \phi$ for any covering isometry $\tilde{h}$ of $f$.

Q.E.D.

By (2) of Lemma 1 and Lemma 2 we have two possibilities as follows

(a) $F(\tilde{f})=\phi$ and $\operatorname{Crit}(\tilde{f}) \neq \phi$

(b) $F(\tilde{f})=\phi$ and so $\operatorname{Crit}(\tilde{f})=F(\tilde{f})$.

Thus in order to get the existence theorem we must control the $F(\tilde{f})$. As to the information of $F(\tilde{f})$ there is the E. Cartan's theorem.

Lemma 3 (E. Cartan) ([2]). Every compact group of isometries of a complete simply connected Riemannian manifold with nonpositive sectional curvature has a fixed point.

Now we say "f-translated geodesic" if an $f$-invariant geodesic is not fixed identically.

Proposition 1. There does not exist a non-trivial f-translated geodesic if the group generated by the covering isometries of $f$ is compact.

Proposition 2. If $\Gamma_{\tilde{f}} \neq 1$ for some covering isometry $\tilde{f}$ where $\Gamma_{\tilde{f}}=$ $\{\mu \in \Gamma \mid \mu \circ \tilde{f}=\tilde{f} \circ \mu\}$, there exists a non-trivial f-translated geodesic or a nontrivial f-fixed geodesic.

Proof. First of all we note the known result that $F(\tilde{f})$ is connected ([2]).

Proof of Proposition 1. If there is a non-trivial $f$-translated geodesic $c$, then we have a non-trivial $\tilde{f}$-invariant geodesic $\tilde{c}$ covering $c$ for some covering isometry $\tilde{f}$. If $\tilde{c}$ is not identically fixed by $\tilde{f}, \tilde{c}(0)=x \in \operatorname{Crit}(\tilde{f})$ - $F(\tilde{f})$ which contradicts (2) of Lemma 1 because $F(\tilde{f}) \neq \phi$ by Lemma 3 . Since $F(\tilde{f})$ is connected there are only identically fixed geodesics.

Proof of Proposition 2. By the hypothesis there is a non-trivial $\mu \in \Gamma_{\tilde{f}}$ such that $\mu \circ \tilde{f} \circ \mu^{-1}=\tilde{f}$. Suppose that $\tilde{f}$ has a fixed point $p$, then $\mu(p)=q$ is also a fixed point of $\tilde{f}$ because $\tilde{f}(q)=\mu \circ \tilde{f} \circ \mu^{-1}(\mu(p))=\mu(p)=q$. Since $\mu$ has no fixed point, $q$ is distinct from $p$. If $\sharp F(f)<\infty$, then this is impossible because $F(\tilde{f})$ is connected. Thus by Lemma 2 we have the 
case (a) which implies the existence of a non-trivial $f$-translated geodesic by (1) of Lemma 1 . The other case is $\operatorname{dim} F(f) \geqq 1$ and so there exists a non-trivial $f$-fixed geodesic.

Q.E.D.

In the proof of Proposition 2 we see the following fact

Corollary 1. Suppose $\sharp F(f)<\infty$, then $F(\tilde{f})=\phi$ if $\Gamma_{\tilde{f}} \neq 1$.

Proposition 3. Let $f$ be an isometry of $M$ which is homotopic to the identity. Then we have (1) there exists a covering isometry $\tilde{f}$ such that it is homotopic to the identity and $d_{f}(x)=d(x, \tilde{f}(x))$ is constant and (2) $f$ has no fixed point with $0<\# F(f)<\infty$.

Corollary 2. If $f$ is homotopic to the identity, then there exists a nontrivial f-translated geodesic or a non-trivial f-fixed geodesic. (This has been proved by Grove [6] in more general case.)

Proof. We prove Proposition 3.

By the covering homotopy property we can take a covering homotopy $H: I \times \tilde{M} \rightarrow \tilde{M}$ such that $H(0, \cdot)=\mathrm{id}_{\tilde{M}}$ and $H(1, \cdot)=\tilde{f}$ with $\pi \circ \tilde{f}=f \circ \pi$. Then we can see that $\tilde{f}$ commutes with any $\gamma \in \Gamma$. Consider $\left(\gamma \circ H_{t}\right)(x)$, $\left(H_{t} \circ \gamma\right)(x)$ for any $x \in \tilde{M}$, then these are paths starting at $\gamma(x)$. Since $\pi\left(\gamma \circ H_{t}\right)=\pi\left(H_{t} \circ \gamma\right)=F_{t} \circ \pi$ where $F_{t}$ is the homotopy connecting $\operatorname{id}_{M}$ and $f$, these paths are the lifts of $\left(F_{t} \circ \pi\right)(x)$ at the same starting point $r(x)$. By the uniqueness of lifting we have $\gamma \circ H_{t}=H_{t} \circ \gamma$ for any $(t, x) \in I \times \tilde{M}$ which implies $\gamma \circ \tilde{f}=\tilde{f} \circ \gamma$. We show $d_{\tilde{f}}=$ constant for this $\tilde{f}$. Since $\tilde{M}$ is a covering space on which $\Gamma$ acts as the covering transformation, there is a fundamental domain $V$ such that $\bar{V}$ is compact and there is $\alpha \in \Gamma$ with $\alpha(x) \in \bar{V}$ for each $x \in \tilde{M}$. By the commutativity $d(x, \tilde{f}(x))=d(\alpha(x)$, $\alpha(\tilde{f}(x)))=d(\alpha(x), \tilde{f}(\alpha(x)))$ and thus we have $d_{\tilde{f}}(x)<\infty$ for any $x \in \tilde{M}$ because of the compactness of $\bar{V}$. It is known that $d_{\tilde{f}}$ is a convex function and so $d_{\tilde{f}}$ is constant.

Next we prove the second part. As seeing in the proof of (1) there is $\tilde{f}$ such that $\tilde{f}$ commutes with $\Gamma$. Since any covering isometry $\tilde{h}$ of $f$ is obtained by $\tilde{h}=\alpha \circ \tilde{f}$ for some $\alpha \in \Gamma$, we have $\Gamma_{\tilde{h}} \neq 1$ which implies $F(\tilde{h})=\phi$ by Corollary 1. However it is impossible because there exists at least one covering isometry $\tilde{g}$ with $F(\tilde{g}) \neq \phi$.

Proof of Corollary 2. In the proof of (1) there is $\tilde{f}$ such that $\tilde{f}$ commutes with $\Gamma$ and so $\Gamma_{\tilde{f}} \neq 1$. We have the conclusion by Proposition 2 .

Q.E.D. 
As looking in the introduction the existence problem is essential in the case of $\# F(f)<\infty$. From now on we fix our consideration in this case. If $F(f)=\left\{p_{1}, p_{2}, \cdots, p_{r}\right\}$, then there is a covering isometry $\tilde{f}_{i}$ such that it has a fixed point on $\pi^{-1}\left(p_{i}\right)$. Thus we consider the following covering isometry class

$$
G_{p_{i}}=\left\{\mu \circ \tilde{f}_{i} \circ \mu^{-1} \mid \mu \in \Gamma\right\} .
$$

Lemma 4. A covering isometry $\tilde{g}$ of $f$ has a fixed point if and only if $\tilde{g} \in G_{p_{i}}$ for some $i$.

Proof. The fixed point of $\tilde{g}$ must be in $\pi^{-1}\left(p_{i}\right)$ for some $i$, now let it be $x$. If $y$ is a fixed point of $\tilde{f}_{i}, \tilde{h}=\gamma \circ \tilde{f}_{i} \circ \gamma^{-1}$ is a covering isometry with the fixed point $x$ for $\gamma \in \Gamma$ such that $\gamma(y)=x$. Thus $\tilde{g}$ and $\tilde{h}$ has the same fixed point and so $\tilde{g}=\tilde{h}$ by the uniqueness of covering. Therefore we have $\tilde{g} \in G_{p_{i}}$. Conversely it is almost same way to show that every element of $G_{p_{i}}$ for any $i$ has a fixed point.

Q.E.D.

Lemma 5. Let $f$ be an isometry of finite order $k(\geqq 1)$. Then a covering isometry $\tilde{f}$ of $f$ has a fixed point if and only if $\tilde{f}^{k}=1$. (It is not necessary the assumption of the finiteness of $F(f)$.)

Proof. Suppose $\tilde{f}$ has a fixed point but $\tilde{f}^{k}=\gamma \neq 1$. Let $x$ be a fixed point of $\tilde{f}$, then $x$ is also a fixed point of $\tilde{f}^{k}$ and so it is a fixed point of $\gamma$. However this is impossible because $\gamma$ is fixed point free. Conversely if $\tilde{f}^{k}=1, \tilde{f}$ has a fixed point by Lemma 3 .

Q.E.D.

Thus we can set up the existence criterion as follows

Existence Criterion Theorem. Let $f$ be an isometry of a nonpositively curved compact manifold $M$ such that $f^{k}=1$ for some integer $k \geqq 1$ and $F(f)=\left\{p_{1}, p_{2}, \cdots, p_{r}\right\}$, then the following statements are mutually equivalent.

(1) There does not exist a non-trivial f-invariant geodesic.

(2) $\tilde{f}^{k}=1$ for any covering isometry $\tilde{f}$ of $f$.

(3) The set of covering isometries of $\tilde{f}$ is just $\bigcup_{i=1}^{r} G_{p_{i}}$.

In particular assume only the finiteness of the fixed point of $f$, then (1) and (3) are equivalent.

Proof. (1) $\Rightarrow(2)$. Suppose that there is a covering $\tilde{f}$ with $\tilde{f}^{k}=1$, then $\tilde{f}$ has no fixed point by Lemma 5 and so the case (a) occurs which implies the existence of a non-trivial $f$-invariant geodesic. This is impossible. 
$(2) \Rightarrow(3)$. If there is a covering $\tilde{f}$ such that $\tilde{f} \oplus \bigcup_{i=1}^{r} G_{p_{i}}, \tilde{f}$ has no fixed point by Lemma 4 . However it is impossible by Lemma 5 .

$(3) \Rightarrow(1)$. Suppose that there exists a non-trivial $f$-invariant geodesic, then the case (b) occurs by the same argument as Proposition 1. By Lemma 4 it is impossible.

The second part is also same.

Q.E.D.

\section{§2. Structure}

We use here the following notations.

$\operatorname{Geo}(M, f)$ : the set of all $f$-invariant geodesics.

$\mathfrak{g}_{[r]}^{f}$ : the free homotopy class of $\operatorname{Geo}(M, f)$ corresponding to the conjugate class $[\gamma]$ of $\gamma \in \Gamma$.

Then in a natural sense $\operatorname{Geo}(M, f)=\cup_{r} \mathrm{~g}_{[r]}^{f}$ and we introduce it's topology from $\Lambda(M, f)$ which was used in [5], [7]. Here we consider the relation between $\mathfrak{g}_{[r]}^{f}$ and $\operatorname{Crit}(\tilde{f})$.

Let $f$ be an isometry of finite order $k(\geqq 1)$.

$G_{\gamma}$ : the set of covering isometries $\tilde{f}$ with $\tilde{f}^{k}=\gamma(\gamma \in \Gamma)$. Now we define an equivalence relation on $G_{\gamma}$ such that $\tilde{f}$ is equivalent $\tilde{g}$ for any $\tilde{f}, \tilde{g} \in G_{r}$ if and only if there is $\xi \in \Gamma_{r}$ with $\tilde{g}=\xi \circ \tilde{f} \circ \xi^{-1}$. The equivalence class of $\tilde{f}$ is written by $\langle\tilde{f}\rangle$.

The main theorem of this section is the following.

STRUCTURe TheOREM I. Let $f$ be an isometry of finite order $k$ on a nonpositively curved compact manifold $M$.

(1) If $\mathfrak{g}_{[r]}^{f} \neq \phi$ for some $\gamma \in \Gamma$, then $\mathfrak{g}_{[r]}^{f}$ is homeomorphic to $\bigcup_{\langle\tilde{j}\rangle} \operatorname{Crit}(\tilde{f}) / \Gamma_{\tilde{f}}$ where $\tilde{f} \in G_{\gamma}$ and $\Gamma_{\hat{f}}=\{\alpha \in \Gamma \mid \alpha \circ \tilde{f}=\tilde{f} \circ \alpha\}$.

(2) If each Crit (f) is a submanifold without boundary, then the homeomorphism in (1) is a diffeomorphism.

Remark. Since Crit $(\tilde{f})$ is a totally geodesic submanifold with possibly non-smooth boundary (see [8]), $\mathfrak{g}_{[r]}^{f}$ is also a submanifold with boundary by the theorem. In particular the case of (2) implies that $\mathfrak{g}_{[r]}^{f}$ is a differentiable manifold.

Of course it is clear $\mathfrak{g}_{[r]}^{f}=F(f)$ for $\gamma=1$ and the structure of $F(f)$ is well known. Hence we assume $r \neq 1$ from now.

First of all we construct a corresponding $\Phi: \cup \operatorname{Crit}(\tilde{f}) \rightarrow g_{[r]}^{f}$ for a covering isometry $\tilde{f}$ with $\tilde{f}^{k}=\gamma$ as follows, By Lemma 5 and (1) of Lemma 1 there exists an $\tilde{f}$-invariant geodesic $\tilde{c}_{p}\left(p \in \operatorname{Crit}(\tilde{f}), \tilde{c}_{p}(0)=p\right)$ and so we 
put $\Phi(p)=\pi \circ \tilde{c}_{p}=c_{x}\left(x=c_{x}(0)\right)$, then $c_{x}$ is an $f$-invariant geodesic and moreover $c_{x}^{k}$ is a representation of [r] because $\tilde{c}_{p}^{k}$ is $\gamma$-invariant, where $c_{x}^{k}(t)$ $=c_{x}(k t), \tilde{c}_{p}^{k}(t)=\tilde{c}_{v}(k t)$. Then it is easy to see the continuity of $\Phi$.

Lemma 6. For any $\tilde{f}, \tilde{g} \in G_{r}$ we have $\tilde{f} \sim \tilde{g}$ if and only if $\Phi(\operatorname{Crit}(\tilde{f}))$ $=\Phi(\operatorname{Crit}(\tilde{g}))$.

Proof. If $\tilde{f} \sim \tilde{g}$ in $G_{r}$, there is $\xi \in \Gamma_{r}$ with $\tilde{g}=\xi \circ \tilde{f} \circ \xi^{-1}$. For any $c_{x} \in$ $\Phi(\operatorname{Crit}(\tilde{f}))$ there is $p \in \operatorname{Crit}(\tilde{f})$ with $\Phi(p)=c_{x}$ and so there is an $\tilde{f}$-invariant geodesic $\tilde{c}_{p}$ such that $\pi \circ \tilde{c}_{p}=c_{x}$. Then $\xi(p) \in \operatorname{Crit}\left(\xi \circ \tilde{f} \circ \xi^{-1}\right)=\operatorname{Crit}(\tilde{g})$ by (3) of Lemma 1. Thus $\xi\left(\tilde{c}_{p}\right)=\tilde{c}_{\xi(p)}$ is a $\tilde{g}$-invariant geodesic and $\Phi(\xi(p))$ $=\pi \circ \tilde{c}_{\xi(p)}=c_{x}$ which implies $\Phi(\operatorname{Crit}(\tilde{f})) \subset \Phi(\operatorname{Crit}(\tilde{g}))$. By the same argument we have $\Phi(\operatorname{Crit}(\tilde{g})) \subset \Phi(\operatorname{Crit}(\tilde{f}))$ and so $\Phi(\operatorname{Crit}(\tilde{f}))=\Phi(\operatorname{Crit}(\tilde{g}))$. Conversely we have only to show that if $\tilde{f} \not \tilde{g}$ in $G_{r}$, then $\Phi(\operatorname{Crit}(\tilde{f})) \cap$ $\Phi(\operatorname{Crit}(\tilde{g}))=\phi$. If not, there is $c_{x} \in \Phi(\operatorname{Crit}(\tilde{f})) \cap \Phi(\operatorname{Crit}(\tilde{g}))$ such that there are $\tilde{f}$-invariant $\tilde{c}_{p}$ and $\tilde{g}$-invariant $\tilde{c}_{q}$ with $\pi \circ \tilde{c}_{p}=c_{x}=\pi \circ \tilde{c}_{q}$. Then there is $\xi \in \Gamma$ such that $\xi\left(\tilde{c}_{p}\right)$ is a lift of $c_{x}$ through a point $q=\xi(p)$ and thus $\xi\left(\tilde{c}_{p}\right)=\tilde{c}_{q}$ because of the uniqueness of the lifting. On the other hand since $q=\xi(p) \in \operatorname{Crit}\left(\xi \circ \tilde{f} \circ \xi^{-1}\right) \cap \operatorname{Crit}(\tilde{g})$, we have $\left(\xi \circ \tilde{f} \circ \xi^{-1}\right)(q)=\tilde{g}(q)$ and so $\xi \circ \tilde{f} \circ \xi^{-1}=\tilde{g}$ by the uniqueness of covering isometry. And $\xi \circ \gamma \circ \xi^{-1}=$ $\xi \circ \tilde{f}^{k} \circ \xi^{-1}=\left(\xi \circ \tilde{f} \circ \xi^{-1}\right)^{k}=\tilde{g}^{k}=\gamma$ implies $\xi \in \Gamma_{r}$. This contradicts $\tilde{f} \nsim \tilde{g}$.

Q.E.D.

Remark. M. Tanaka kindly notified the author of this lemma and of it's usefulness for proving Structure theorems I, II.

Proof of (1). Now we show that for any $c_{x} \in \mathfrak{g}_{[r]}^{f}$ there are $\tilde{h} \in G_{r}$ and an $\tilde{h}$-invariant geodesic $\tilde{c}_{p}$ with $\pi \circ \tilde{c}_{p}=c_{x}$. Let $\tilde{c}_{q}$ be a lift of $c_{x}$ which is an $\tilde{g}$-invariant geodesic for some covering isometry $\tilde{g}$ with $\tilde{g}^{k}=\eta(\eta \in \Gamma)$. Since $\pi \circ \tilde{c}_{q}=c_{x}$ is an element of $\mathfrak{g}_{[r]}^{f}, \eta \in[\gamma]$ and so $\eta=\xi \circ \gamma \circ \xi^{-1}$ for some $\xi \in \Gamma$. Put $\tilde{h}=\xi^{-1} \circ \tilde{g} \circ \xi$, then $\tilde{h} \in G_{r}$. Now take an $\tilde{h}$-invariant geodesic $\tilde{c}_{\xi^{-1}(q)}$, then $\tilde{c}_{\xi^{-1}(q)}$ is satisfied with $\pi \circ \tilde{c}_{\xi^{-1}(q)}=c_{x}$. Finally by this fact and Lemma 6 we have the surjection $\Phi: \bigcup_{\langle\tilde{f}\rangle} \operatorname{Crit}(\tilde{f}) \rightarrow \mathfrak{g}_{[r]}^{f}$. Next we show that if $\Phi(p)=\Phi(q)$ for any point $p \neq q \in \operatorname{Crit}(\tilde{f})$, then there exists $\mu \in \Gamma_{\tilde{f}}$ such that $\mu(p)=q$. Put $c_{x}=\Phi(p)\left(=\Phi(q), x=c_{x}(0)\right)$ and let $\tilde{c}_{p}$, $\tilde{c}_{q}$ are the lifts of $c_{x}\left(\tilde{c}_{p}(0)=p, \tilde{c}_{q}(0)=q\right)$. Since the both lifts are $\tilde{f}$-invariant, these are $\tilde{f}^{k}=\gamma$-invariant. Thus there exists $\mu \in \Gamma_{\gamma}$ such that $\mu(p)=q$ by the fact discussed in [10]. By the way $\tilde{f}^{-1} \circ \mu \circ \tilde{f}=\xi$ for some $\xi$ because the covering isometry normalizes $\Gamma$. Since $\mu \circ \tilde{f}(p)=\tilde{f} \circ \mu(p)$ at $p$, we have 
$\xi(p)=\mu(p)$ and so $\xi=\mu$ because $\Gamma$ has no fixed point. Hence $\tilde{f} \circ \mu=\mu \circ \tilde{f}$ and $\Phi$ induce the one to one corresponding $\bar{\Phi}: \bigcup_{\langle\tilde{f}\rangle} \operatorname{Crit}(\tilde{f}) / \Gamma_{\tilde{f}} \rightarrow \mathfrak{g}_{[r]}^{f}$. The continuity of $\bar{\Phi}$ and $\bar{\Phi}^{-1}$ is clear from the construction.

Q.E.D.

For the proof of (2) we need some lemmas. The next lemma is proved as same as Theorem 1.3.8 in [8] and so we omit here the proof.

Lemma 7. Suppose that Crit $(\tilde{f})$ satisfies the assumption of (2) in the theorem. If $\tilde{X}_{p}$ is a tangent vector of $\operatorname{Crit}(\tilde{f})$ at $p$ which is transversal to the $\tilde{f}$-invariant geodesic $\tilde{c}_{p}$, then the surface $H: R \times(-\varepsilon, \varepsilon) \rightarrow$ Crit $(\tilde{f})$ defined by $H(s, t)=\tilde{c}_{\exp \left(t \tilde{X}_{p}\right)}(s)$ is totally geodesic and it's curvature is zero.

Lemma 8. Suppose that Crit $(\tilde{f})$ satisfies the assumption of (2) in the theorem. Then $\Phi: \operatorname{Crit}(\tilde{f}) \rightarrow \Lambda(M, f)$ is a smooth immersion.

Proof. $\Phi$ is a composition map of $\theta: \operatorname{Crit}(\tilde{f}) \rightarrow \Lambda(\tilde{M}, \tilde{f})$ and $\pi_{\sharp}: \Lambda(\tilde{M}, \tilde{f})$ $\rightarrow \Lambda(M, f)$ defined $\pi_{\sharp} \circ \tilde{c}(t)=\pi(\tilde{c}(t))$. At first we show $\theta$ is smooth. Let $\theta(p)$ $=\tilde{c}_{p}$, then we have only to prove $\operatorname{Exp}^{-1} \circ \theta \circ \widetilde{\exp }$ is smooth where $\left(U_{p}, \widetilde{\exp }^{-1}\right)$ and $\left(V_{\tilde{c}_{p}}, \operatorname{Exp}^{-1}\right)$ are local charts of $p$ and $\tilde{c}_{p}$. For any $\tilde{X}_{p} \in T_{p}(\operatorname{Crit}(\tilde{f}))$ with $\widetilde{\exp }\left(\tilde{X}_{p}\right) \in U_{p}, \quad \operatorname{Exp}^{-1} \circ \theta \circ \widetilde{\exp }\left(\tilde{X}_{p}\right)=\operatorname{Exp}^{-1} \circ \theta(q)=\operatorname{Exp}^{-1}\left(\tilde{c}_{q}\right)=\tilde{X}$ is $H_{*}(\partial / \partial t)(s, t)$ by using Lemma 7 and moreover $\tilde{X}=H_{*}(\partial / \partial t)(s, 0)$ is parallel along $\tilde{c}_{p}$ and $\tilde{X}(p)=\tilde{X}_{p}$. Since $\tilde{X}$ is parallel, it is determined uniquely by $\tilde{X}_{p}$ and $\operatorname{Exp}^{-1} \circ \theta \circ \widetilde{\exp :} \tilde{X}_{p} \rightarrow \tilde{X}$ is linear injective which implies $\theta$ is smooth and $\theta_{*}$ is injective. Consequently $\Phi=\pi_{\sharp} \circ \theta$ is smooth because $\pi$ is a covering map. Moreover by the injectivity of $\theta_{*}$ and the uniqueness of lift we have $\Phi$ is also a smooth immersion.

Q.E.D.

Proof of (2). Since the smooth immersion $\Phi$ in Lemma 8 is actually into $\mathfrak{g}_{[r]}^{f}$ and $\bigcup_{\langle\hat{f}\rangle} \operatorname{Crit}(\tilde{f}) / \Gamma_{\tilde{f}} \cong \mathfrak{g}_{[r]}^{f}$ by (1), $\bar{\Phi}: \bigcup_{\langle\tilde{f}\rangle} \operatorname{Crit}(\tilde{f}) / \Gamma_{\tilde{f}} \rightarrow \mathfrak{g}_{[r]}^{f} \subset \Lambda(M, f)$ is a smooth embedding. Thus $\mathfrak{g}_{[r]}^{f}$ becomes a submanifold of $\Lambda(M, f)$ which implies that $\bar{\Phi}$ is a diffeomorphism.

Q.E.D.

Remark. If $M$ is a locally symmetric space of nonpositively curved, then each $\operatorname{Crit}(\tilde{f})$ is an analytic submanifold without boundary. And in this case $\operatorname{Crit}(\tilde{f})=Z_{I(\tilde{\mu})}^{0}(\tilde{f}) \cdot x$ for $x \in \operatorname{Crit}(\tilde{f})$ where $Z_{I(\tilde{H})}^{0}(\tilde{f})$ is the identity component of centralizer of $\tilde{f}$ in $I(\tilde{M})$ and $I(\tilde{M})$ is the group of isometries of $\tilde{M}([8])$.

Until now our interest was "closed geodesics". However, the above consideration is valid for a general isometry. And so we note the similar structure theorem which was suggested by M. Tanaka. 
Let $G_{f}$ be the set of all covering isometries of $f$, then we define an equivalence relation in $G_{f}$ as follows, $\tilde{f} \sim \tilde{g}$ for any $\tilde{f}, \tilde{g} \in G_{f}$ if and only if there is $\xi \in \Gamma$ such that $\tilde{g}=\xi \circ \tilde{f} \circ \xi^{-1}$. Put $g[\tilde{f}]=\{c \in \operatorname{Geo}(M, f) \mid$ Each lift of $c$ is $\xi \circ \tilde{f} \circ \xi^{-1}$-invariant for some $\left.\xi \in \Gamma\right\}$, then the corresponding $\Phi: \operatorname{Crit}(\tilde{f})$ $\rightarrow \mathfrak{g}[\tilde{f}]$ is defined also as above. Now our statement is the following

Structure Theorem II. Let $f$ be an isometry on a compact nonpositively curved manifold. Then $\bar{\Phi}: \bigcup_{\langle\tilde{f}\rangle} \operatorname{Crit}(\tilde{f}) / \Gamma_{\tilde{f}} \rightarrow \mathfrak{g}[\tilde{f}]$ is a homeomorphism and moreover it is a diffeomorphism if each $\operatorname{Crit}(\tilde{f})$ is a submanifold without boundary.

\section{§3. Example}

Let $M$ be a flat torus.

(1) $f=\pi / 2$ rotation. Assume the fig. 1 as $M$. Then $F(f)=\left\{p_{1}, p_{2}\right\}$, $f^{4}=1$ and $\pi_{1}(M)=Z \times Z$.

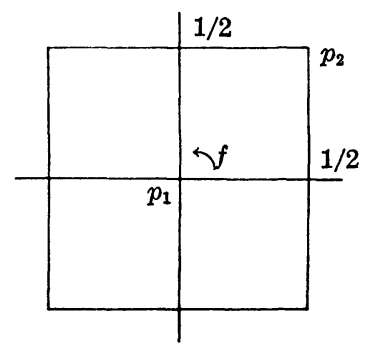

fig. 1

Any covering isometry $\tilde{f}$ of $f$ is

$$
\tilde{f}(x)=\left[\begin{array}{rr}
0 & -1 \\
1 & 0
\end{array}\right] \cdot x+\left[\begin{array}{c}
m \\
n
\end{array}\right] \quad \text { for } x \in R^{2}=\tilde{M}
$$

where $m, n \in Z$. It is easy to check $\tilde{f}^{4}=1$ which implies that there does not exist a nontrivial $f$-invariant geodesic by our theorem. In this case the set $G$ of the covering isometries is as follows, $G=G_{p_{1}} \cup G_{p_{2}}$

$$
\begin{aligned}
& G_{p_{1}}=\left\{\left[\begin{array}{rr}
0 & -1 \\
1 & 0
\end{array}\right] \cdot x+\left[\begin{array}{c}
m \\
n
\end{array}\right] \mid m \equiv n(2)\right\}=\left\{\mu \circ \tilde{f}_{1} \circ \mu^{-1} \mid \mu \in \Gamma\right\} \\
& G_{p_{2}}=\left\{\left[\begin{array}{rr}
0 & -1 \\
1 & 0
\end{array}\right] \cdot x+\left[\begin{array}{c}
m \\
n
\end{array}\right] \mid m+n \equiv 1(2)\right\}=\left\{\mu \circ \tilde{f}_{2} \circ \mu^{-1} \mid \mu \in \Gamma\right\}
\end{aligned}
$$

where $\tilde{f}_{1}(x)=\left[\begin{array}{rr}0 & -1 \\ 1 & 0\end{array}\right] \cdot x, \tilde{f}_{2}(x)=\left[\begin{array}{rr}0 & -1 \\ 1 & 0\end{array}\right] \cdot x+\left[\begin{array}{l}1 \\ 0\end{array}\right]$ and $\Gamma_{\tilde{f}_{1}}=\Gamma_{\tilde{f}_{2}}=\{\mathrm{id}\}$. 
(2) $f=$ the reflection with respect to $x$-axis.

$$
\begin{array}{ll}
F(f)=X_{1} \cup X_{2}, & X_{1}=\left\{\left[\begin{array}{l}
x \\
0
\end{array}\right] \mid-1 / 2 \leqq x \leqq 1 / 2\right\} \text { and } \\
X_{2}=\left\{\left[\begin{array}{c}
x \\
\pm 1 / 2
\end{array}\right] \mid-1 / 2 \leqq x \leqq 1 / 2\right\} \\
f^{2}=1
\end{array}
$$

Any covering isometry $\tilde{f}$ of $f$ is

$$
\tilde{f}(x)=\left[\begin{array}{rr}
1 & 0 \\
0 & -1
\end{array}\right] \cdot x+\left[\begin{array}{c}
m \\
n
\end{array}\right] \quad x \in R^{2}=\tilde{M} \quad \text { where } \quad m, n \in Z .
$$

Now we see the property of the covering isometries. In this case $m=0$ if and only if $\tilde{f}$ has a fixed point, and so the class $G_{\text {id }}$ have the fixed point.

$$
\begin{aligned}
& G_{\mathrm{id}}=G_{1}^{\mathrm{id}} \cup G_{2}^{\mathrm{id}} \\
& G_{1}^{\mathrm{id}}=\left\{\mu \circ \tilde{f}_{1} \circ \mu^{-1} \mid \mu \in \Gamma\right\}=\left\{\left[\begin{array}{rr}
1 & 0 \\
0 & -1
\end{array}\right] \cdot x+\left[\begin{array}{l}
0 \\
n
\end{array}\right] \mid n \equiv 0(2)\right\} \\
& G_{2}^{\mathrm{id}}=\left\{\mu \circ \tilde{f}_{2} \circ \mu^{-1} \mid \mu \in \Gamma\right\}=\left\{\left[\begin{array}{rr}
1 & 0 \\
0 & -1
\end{array}\right] \cdot x+\left[\begin{array}{l}
0 \\
n
\end{array}\right] \mid n \equiv 1(2)\right\}
\end{aligned}
$$

where

$$
\begin{aligned}
& \tilde{f}_{1}(x)=\left[\begin{array}{rr}
1 & 0 \\
0 & -1
\end{array}\right] \cdot x, \tilde{f}_{2}(x)=\left[\begin{array}{rr}
1 & 0 \\
0 & -1
\end{array}\right] \cdot x+\left[\begin{array}{l}
0 \\
1
\end{array}\right], \text { and } \\
& \Gamma_{\tilde{f}_{1}}=\Gamma_{\tilde{f}_{2}}=\left\{\mu \in \Gamma \mid \mu(x)=x+\left[\begin{array}{c}
m \\
0
\end{array}\right], m \in Z\right\} .
\end{aligned}
$$

Then $\mathfrak{g}_{[1]}^{f}=\{$ one point invariant geodesics $\}=F(f) \cong \operatorname{Crit}\left(\tilde{f}_{1}\right) / \Gamma_{\tilde{f}_{1}} \cup$ $\operatorname{Crit}\left(\tilde{f}_{2}\right) / \Gamma_{\tilde{f}_{2}}$.

On the other hand if $m \neq 0, \tilde{f}$ has no fixed point and so $\tilde{f}^{2}=\gamma \neq 1$. In this case $\gamma$ is a following form, $\gamma(x)=x+\left[\begin{array}{c}2 m \\ 0\end{array}\right]$. Then $G_{r}$ is

$$
\begin{aligned}
& G_{r}=G_{1}^{r} \cup G_{2}^{r} \\
& G_{1}^{r}=\left\{\mu \circ \tilde{g}_{1} \circ \mu^{-1} \mid \mu \in \Gamma_{r}\right\}=\left\{\left[\begin{array}{rr}
1 & 0 \\
0 & -1
\end{array}\right] \cdot x+\left[\begin{array}{l}
m \\
n
\end{array}\right] \mid n \equiv 0(2)\right\} \\
& G_{2}^{r}=\left\{\mu \circ \tilde{g}_{2} \circ \mu^{-1} \mid \mu \in \Gamma_{\gamma}\right\}=\left\{\left[\begin{array}{rr}
1 & 0 \\
0 & -1
\end{array}\right] \cdot x+\left[\begin{array}{l}
m \\
n
\end{array}\right] \mid n \equiv 1(2)\right\}
\end{aligned}
$$

where 


$$
\tilde{g}_{1}(x)=\left[\begin{array}{rr}
1 & 0 \\
0 & -1
\end{array}\right] \cdot x+\left[\begin{array}{c}
m \\
0
\end{array}\right], \quad \tilde{g}_{2}(x)=\left[\begin{array}{rr}
1 & 0 \\
0 & -1
\end{array}\right] \cdot x+\left[\begin{array}{c}
m \\
1
\end{array}\right] .
$$

The structure theorem implies

$$
\mathfrak{g}_{[\gamma]}^{f} \cong \operatorname{Crit}\left(\tilde{g}_{1}\right) / \Gamma_{\tilde{g}_{1}} \cup \operatorname{Crit}\left(\tilde{g}_{2}\right) / \Gamma_{\tilde{g}_{2}} \quad \text { for the above } \gamma .
$$

More in detail Crit $\left(\tilde{g}_{i}\right)=Z_{E(2)}\left(\tilde{g}_{i}\right) \cdot x$ for $x \in \operatorname{Crit}\left(\tilde{g}_{i}\right)$ where $E(2)=$ Euclidean group of isometries of $R^{2}$ (see Remark in $\S 2$ ). Thus we have

$$
\begin{aligned}
& \operatorname{Crit}\left(\tilde{g}_{i}\right)=\left\{e_{i} \in E(2) \mid e_{i}(x)=x+\left[\begin{array}{c}
r_{i} \\
0
\end{array}\right], r_{i} \in R\right\} \\
& \Gamma_{\tilde{s}_{i}}=\left\{\mu_{i} \in \Gamma \mid \mu_{i}(x)=x+\left[\begin{array}{c}
s_{i} \\
0
\end{array}\right], s_{i} \in Z\right\}
\end{aligned}
$$

and finally Crit $\left(\tilde{g}_{i}\right) / \Gamma_{\tilde{g}_{i}} \cong S^{1}$ (1-dimensional sphere). Therefore we have $\mathfrak{g}_{[r]}^{f} \cong S^{1} \cup S^{1}$.

\section{REFERENCES}

[1] Klingenberg, W., Riemannian Geometry, Walter de Gruyter, 1982.

[2] Kobayashi, S., Transformation groups in differential geometry, Ergeb. der Math., Band, 70 (1972).

[ 3 ] Kurogi, T., Riemannian manifolds admitting some geodesics, Proc. Japan Acad., 50 (1974), 124-126.

[4] —_, Riemannian manifolds admitting some geodesics II, ibid., 52 (1976), 7-9.

[5] Grove, K., Condition (C) for the energy integral on certain path spaces and applications to the theory of geodesics, J. Differential Geom., 8 (1973), 207-223.

[6] —-, Isometry invariant geodesic, Topology, 13 (1974), 281-292.

[ 7 ] Grove, K. and Tanaka, M., On the number of invariant closed geodesics, Acta Math., 140 (1978), 33-48.

[ 8 ] Ozols, V., Critical point of the displacement function of an isometry, J. Differential Geom., 3 (1969), 411-432.

[ 9 ] Sunada, T., Closed geodesics in locally symmetric space, Tohoku Math. J., 30 (1978), 59-68.

[10] - Trace formula and heat equation asymptotic for a nonpositively curved manifold, Amer. J. Math., 103 (1982), 411-435.

[11] Tanaka, M., On the existence of infinitely many isometry invariant geodesics, J. Differential Geom., 17 (1982), 171-184.

[12] - On the number of closed geodesics and isometry invariant geodesics, Preprint of Proceedings of Geodesic at Tokyo University, 1982.

Department of Mathematics

Faculty of Education

Fukui University

Fukui, 910 Japan 